\title{
Quantitative Detection of Trace Level Cloxacillin in Food Samples Using Magnetic Molecularly Imprinted Polymer Extraction and Surface-Enhanced Raman Spectroscopy Nanopillars
}

Ashley, Jon; Wu, Kaiyu; Hansen, Mikkel Fougt; Schmidt, Michael Stenbæk; Boisen, Anja; Sun, Yi

Published in:

Analytical Chemistry

Link to article, DOI:

10.1021/acs.analchem.7b02725

Publication date:

2017

Document Version

Peer reviewed version

Link back to DTU Orbit

Citation (APA):

Ashley, J., Wu, K., Hansen, M. F., Schmidt, M. S., Boisen, A., \& Sun, Y. (2017). Quantitative Detection of Trace Level Cloxacillin in Food Samples Using Magnetic Molecularly Imprinted Polymer Extraction and SurfaceEnhanced Raman Spectroscopy Nanopillars. Analytical Chemistry, 89(21), 11484-11490.

https://doi.org/10.1021/acs.analchem.7b02725

\section{General rights}

Copyright and moral rights for the publications made accessible in the public portal are retained by the authors and/or other copyright owners and it is a condition of accessing publications that users recognise and abide by the legal requirements associated with these rights.

- Users may download and print one copy of any publication from the public portal for the purpose of private study or research.

- You may not further distribute the material or use it for any profit-making activity or commercial gain

- You may freely distribute the URL identifying the publication in the public portal 


\title{
Rapid and Quantitative Detection of Trace Level Cloxacillin in Food Samples Using Magnetic MIP Extraction and SERS Nanopil- lars
}

\author{
Jon Ashley, ${ }^{\ddagger \dagger}$ Kaiyu Wu,${ }^{\dagger, \dagger, \S, *}$ Mikkel Fougt Hansen, ${ }^{\dagger}$ Michael Stenbæk Schmidt, ${ }^{\dagger, \S}$ Anja Boisen, ${ }^{\dagger, \S}$ \\ Yi Sun ${ }^{\dagger} * *$ \\ $\dagger$ DTU Nanotech, Department of Micro- and Nanotechnology, Technical University of Denmark, Ørsteds Plads, DK- \\ 2800 Kgs, Lyngby, Denmark \\ $\S$ DNRF and Villum Fonden Center for Intelligent Drug Delivery and Sensing Using Microcontainers and Nanomechan- \\ ics, IDUN, Ørsteds Plads, DK-2800 Kgs, Lyngby, Denmark
}

\begin{abstract}
There is an increasing demand for rapid, sensitive and low cost analytical methods to routinely screen antibiotic residues in food products. Conventional detection of antibiotics involves sample preparation by liquid-liquid or solid phase extraction, followed by analysis using LC-MS, CE or GC. The process is labor-intensive, time-consuming and expensive. In this study, we developed a new analytical method that combines magnetic molecularly imprinted polymer (MMIP)-based sample preparation with surface-enhanced Raman spectroscopy (SERS)-based detection for quantitative analysis of cloxacillin in pig serum. MMIP microspheres were synthesized using core-shell technique. The large loading capacity and high selectivity of the MMIP microspheres enabled efficient extraction of cloxacillin, while the magnetically susceptible characteristics greatly simplified sample handling procedures. Low cost and robust SERS substrates consisting of vertical gold capped silicon nanopillars were fabricated and employed for the detection of cloxacillin. Quantitative SERS was achieved by normalizing signal intensities using an internal standard. By coherently combining MMIP extraction and silicon nanopillar-based SERS biosensor, good sensitivity towards cloxacillin was achieved. The detection limit was 7.8 pmol. Cloxacillin recoveries from spiked pig plasma samples were found to be more than $80 \%$.
\end{abstract}


There is an increasing concern over the widespread use of antibiotic drugs in animal agriculture. Drug residues can accumulate in human bodies through the food chain, causing various side effects. Moreover, the use of antibiotics has caused the spread of antibiotics resistance in farms, and the transmission of resistant bacteria of animal origin to humans has become a serious health threat. Cloxacillin is among the most commonly prescribed antibiotics in veterinary practices. It has been used as prophylactic/therapeutic agents or as feed additives for growth promotion. Routine screening of cloxacillin is required for a number of food matrices. According to the EU commission regulation No 37/2010, the maximum residue limits (MRLs) of cloxacillin is $300 \mu \mathrm{g} / \mathrm{kg}$ in muscle, fat, liver and kidney, and $30 \mu \mathrm{g} / \mathrm{kg}$ in milk $^{1}$.

To accurately quantify the trace amount of cloxacillin in complex food matrices, efficient sample preparation and analytical methods are equally important. Conventional sample preparation methods, such as liquid-liquid extraction, solid-phase extraction (SPE) or solid-phase micro extraction (SPME) are well established, but these techniques are labor-intensive, time-consuming and limited by poor selectivity, which makes sample preparation a major bottleneck in the analytical process $^{2}$. For the detection of cloxacillin, classical analytical techniques including high performance liquid chromatography (HPLC), liquid Chromatography-mass spectrometry (LC-MS), gas chromatography-mass spectrometry (GC-MS) are generally used due to their excellent sensitivity and precision ${ }^{3-5}$. However, these methods require expensive instrumentation and long analysis procedures. As such, in order to rapidly, accurately and economically screen the cloxacillin residues in food matrices, both new sample preparation methods and new analytical methods need to be developed ${ }^{6}$.

Recently, molecularly imprinted polymers (MIPs), synthetic materials with recognition sites that can specifically bind to target molecules, have attracted immense attention for use in sample preparation. Compared to conventional sorbents e.g. C18 silica gel, MIPs offer apparent advantages such as higher loading capacities and enhanced selectivity, allowing for improved sample recovery. MIPs have been employed as sorbents in solid-phase extraction to extract pesticides, antibiotics or growth hormones from milk, juice and meat products $^{10}$. In addition, MIP technology can also be combined with magnetic nanoparticles, resulting in magnetic MIPs (MMIPs) which possess both magnetically susceptible characteristics and good selectivity for the target molecules ${ }^{7}$. The MMIPs could significantly simplify sample handling and pre-treatment procedures, and thus hold great potential for facile sample preparation at the point of source. So far, MIPs specific to cloxacillin have been synthesized and applied in classical SPE and $\mathrm{SPME}^{8,9}$. However, to the best of our knowledge, the use of MMIPs to directly extract cloxacillin from food samples has seldom been demonstrated.

Surface-enhanced Raman spectroscopy (SERS) is a powerful analytical method, which uses nanoplasmonic hot spots to magnify chemical information of analytes adsorbed to or near the surface of noble metals ${ }^{10,11}$. SERS biosensors have demonstrated an ability to detect small molecule analytes with high sensitivity and short analysis times ${ }^{12}$. However, the use of SERS biosensors in real-world applications is greatly hindered due to the lack of reliable and cost-effective SERS sub- strates $^{13}$. Many of the reported SERS substrates are colloidbased ${ }^{14-19}$ and suffer from inconsistent performance. Previously, we have developed wafer-scale metal capped silicon nanopillars with a high aspect ratio as SERS substrates ${ }^{20-22}$. The key advantages of such SERS substrates are their simple fabrication process and signal uniformity. The low fabrication cost and the high integrity of the SERS substrate make this biosensor very attractive for routine analysis. Besides the substrate, another challenge associated with SERS is the background interference, since background interference can seriously affect the quantification of trace analytes. Consequently, sample pretreatment is generally required to reduce or remove undesirable interference before SERS detection ${ }^{23}$. As such, a combination of the SERS biosensor with a simple sample preparation technique would provide a fast and sensitive analytical method for detection of drug residues from complex food components.

In this paper, we innovatively integrated MMIP-based extraction with SERS-based detection for the quantitative analysis of cloxacillin in pig serum. The MMIP microspheres were synthesized using the core-shell technique, where superparamagnetic iron oxide microspheres were formed as the core, and a MIP layer for capturing cloxacillin was deposited using free radical polymerization. The SERS substrate consisting of metal-capped silicon nanopillars was fabricated by a lithography-free reactive ion etching process followed by e-beam assisted metal evaporation. Cloxacillin in pig serum was extracted using the MMIP microspheres, and the eluted analyte was directly dried on the SERS substrate for detection. The whole process could in principle be finished within 1 hour. The biosensor achieved good sensitivity towards cloxacillin with a limit of detection (LOD) of 7.8 pmol. Recoveries of spiked cloxacillin in porcine plasma ranged from 85 to $126 \%$. The developed analytical method could potentially be integrated onto a miniaturized instrumentation, enabling the analysis to be performed outside a laboratory setting.

\section{EXPERIMENTAL SECTION}

Materials and procedures. Ferric chloride $\mathrm{FeCl} 3 \cdot 6 \mathrm{H} 2 \mathrm{O}$, ethylene glycol, sodium acetate, polyethylene glycol (PEG) MW 2000, tetraethoxysilane (TEOS), 35\% ammonium hydroxide, metacryloxy propyl tri methoxyl silane (MPA), cloxacillin, ethylene glycol dimethacrylate (EDGMA), meth acrylic acid (MAA), N-isopropylacrylamide (NIPAm) and azo(bis)isobutyronitrile (AIBN) were purchased from Sigma Aldrich. Inhibitors were removed from all monomers prior to polymerization using pre-packed columns from Sigma Aldrich. All solvents were at analytical grade and used without further purification.

Synthesis of MMIP microspheres. The MMIP microspheres were synthesized using the core-shell technique. Firstly, iron oxide $\left(\mathrm{FeO}_{\mathrm{x}}\right)$ microspheres were synthesized as the magnetic core using the thermal solvent method. Briefly, $\mathrm{FeCl}_{3} \cdot 6 \mathrm{H}_{2} \mathrm{O}$ $(1.35 \mathrm{~g})$ was dissolved in ethylene glycol $(40 \mathrm{ml})$. Anhydrous sodium acetate (3.6 g), and PEG MW 2000 (0.1 g) were added sequentially and the mixture was stirred for 30 minutes to form a homogenous orange suspension. The solution was then degassed under vacuum for 30 minutes, transferred to a 100 
$\mathrm{ml}$ autoclave reactor and heated in a furnace oven at $200{ }^{\circ} \mathrm{C}$ for 10 hours. The resultant black precipitate was washed several times with ethanol and dried under vacuum for 6 hours. Next, a $\mathrm{SiO}_{2}$ layer was added to the magnetic core. 0.5 $\mathrm{g}$ of the resultant $\mathrm{FeO}_{\mathrm{x}}$ microspheres was re-suspended in ethanol $(60 \mathrm{ml})$ and water $(12 \mathrm{ml})$. The black solution was then sonicated using a probe sonicator for at least 15 minutes followed by the addition of 35\% ammonia hydroxide $(2 \mathrm{ml})$, TEOS $(2 \mathrm{ml})$. The solution was allowed to react under pulse sonication for 4 hours before transferring to an orbital shaker. The resultant particles were washed several times with water until the $\mathrm{pH}$ had returned to neutral. This was followed by washing with ethanol several times and dried under vacuum for 12 hours. After depositing the $\mathrm{SiO}_{2}$ layer, the microsphere surface was modified with MPA to introduce a polymerizable group to the surface. The $\mathrm{FeO}_{\mathrm{x}} @ \mathrm{SiO}_{2}$ microspheres $(0.4 \mathrm{~g})$ were mixed with 4\% (v:v) MPA: anhydrous toluene. The solution was sonicated for 15 minutes, followed by heating to $60{ }^{\circ} \mathrm{C}$ and overhead stirring overnight under nitrogen. The $\mathrm{FeO}_{\mathrm{x}} @ \mathrm{SiO}_{2}-\mathrm{MPA}$ microspheres were washed several times using ethanol before drying for 10 hours under vacuum.

The last step was to graft the MIP layer onto the core. A solution containing, MAA (2 mmol), EDGMA (2 mmol), NIPAm (4 mmol), cloxacillin $(1 \mathrm{mmol})$ in methanol $(60 \mathrm{ml})$ was prepared and degassed by bubbling nitrogen for 30 minutes. The solution was then added to a three way round bottom flask containing $0.4 \mathrm{~g}$ of $\mathrm{FeO}_{\mathrm{x}} @ \mathrm{SiO}_{2}-\mathrm{MPA}$ microspheres and stirred for an hour at room temperature. The polymerization reaction was initiated by adding AIBN (40mg) and heating the mixture to $60{ }^{\circ} \mathrm{C}$ for 16 hours under nitrogen protection. The resultant MMIP microspheres were transferred to a Soxhlet extractor and continuously washed using 9:1 (v:v) methanol:acetic acid for 24 hours to remove the template. The washed microspheres were then recovered by sonicating the thimbles in methanol. The microspheres were finally washed several times with methanol to remove residual acetic acid and dried under vacuum. Magnetic non-imprinted polymer (MNIP) microspheres were prepared in the same manner as for MMIP microspheres in the absence of the template.

Characterization of MMIP microspheres. X-ray diffraction experiments were performed on a Bruker D8 FOCUS x-ray diffractometer. IR spectra were taken using a Spectrum 100 (Perkin Elmer, MA, USA). All UV absorbance measurements were done on a UV-2600 (Shimazu, Kyoto Japan). All SEM images and ESR images were taken using a Quanta FEG SEM (FEI, Oregon USA). TEM images were taken using a Tecnai T20 G2 (FEI, Oregon USA) transmission electron microscope. All VSM hysteresis loops were measured on a LakeShore model 7407 (Lakeshore Crytronics, OH, USA) vibrating sample magnetometer (VSM). A Thermo Scientific DXRxi Raman imaging microscope was used for all SERS measurements.

Binding capacity and binding kinetics. The binding capacity and binding kinetics of cloxacillin to both the MMIP and MNIP microspheres were measured using UV absorbance. For the binding capacity, $10 \mathrm{ml}$ samples containing $0-125$ $\mu \mathrm{M}$ of cloxacillin were incubated with $10 \mathrm{mg}$ of MMIP and MNIP microspheres respectively for 12 hours. The binding capacities were determined by calculating the difference in
UV absorbance at $220 \mathrm{~nm}$ before and after incubation (see supplementary information). To determine the binding kinetics, $10 \mathrm{ml}$ samples containing the optimum concentration of analyte determined from the binding capacity was incubated with $10 \mathrm{mg}$ of MMIP and MNIP microspheres for different time period ranging from 1 minute to 2 hours. The samples were centrifuged for 15 minutes at $14000 \times \mathrm{g}$. The binding kinetics assays were determined again by the change in absorbance at $220 \mathrm{~nm}$ before and after incubation. The selectivity was determined by incubating MMIP and MNIP microspheres, respectively, with $100 \mu \mathrm{M}$ of deoxycycline, spiramycin and gentamycin for 12 hours with shaking at room temperature. The response of each antibiotic was determined from the change in absorbance before and after incubation.

Fabrication of the SERS substrate. Vertically standing nanopillars were fabricated by a lithography-free process. Starting from a 4-inch Si wafer, a maskless reactive ion etching process was employed to form silicon nanopillars with radius $\approx 20 \pm 4 \mathrm{~nm}$. P-type single side (100) polished wafers were used (Topsil). Maskless reactive ion etching was performed in an advanced silicon etcher (Surface Technology Systems MESC Multiplex ICP) at an $\mathrm{SF}_{6}: \mathrm{O}_{2}$ flow ratio of 1.12 , a platen power of $120 \mathrm{~W}$, and a chamber pressure of 36 mTorr. The employed etching time was 4 minutes. Silicon nanopillars were formed at a rate of $\sim 3 \mathrm{~nm} / \mathrm{s} . \mathrm{O}_{2}$ plasma treatment was then applied to remove etching by-products from the surface of the silicon nanopillars. An $\mathrm{O}_{2}$ flow of 45 sccm, a platen power of $20 \mathrm{~W}$, a coil power of $800 \mathrm{~W}$, and a chamber pressure of 10 mTorr were used. The time of the $\mathrm{O}_{2}$ plasma treatment was 30 seconds. Lastly, a $225 \mathrm{~nm}$ thick gold film was deposited onto the silicon nanopillars by e-beam evaporation. A Wordentec QCL 800 was used for the deposition at a pressure of $\sim 4 \times 10^{-6} \mathrm{mbar}$, with a deposition rate of $10 \AA / \mathrm{s}$. The deposition resulted in the formation of gold caps at the apex of the silicon nanopillars, along with a gold film on the underlying silicon surface. The manufactured substrates were stored in a vacuum (Thermo Scientific Nalgene desiccator) to minimize degradation.

SERS Detection. A Thermo Scientific DXRxi Raman imaging microscope was used for all SERS measurements. The microscope was coupled to a single grating spectrometer with a $5 \mathrm{~cm}^{-1}$ FWHM spectral resolution and a \pm 2 wavenumber accuracy. All measurements were conducted at room temperatures with a $50 \mu \mathrm{m}$ slit and a $10 \times$ objective lens. An excitation wavelength of $780 \mathrm{~nm}$ was employed with a spot size of $\sim 3.1$ $\mu \mathrm{m}$ in diameter. The laser power was $10 \mathrm{~mW}$. The signal acquisition time was $50 \mathrm{~ms}$. The electron multiplying feature of the charge-coupled device was switched off. A step size of $100 \mu \mathrm{m}$ was used for SERS imaging. $1 \mu \mathrm{l}$ of cloxacillin standard (5 - $1000 \mathrm{pmol})$ were dried on each substrate. The ratio of the intensity of the peaks shown at 931 and $1034 \mathrm{~cm}^{-1}$ was averaged over a $1 \mathrm{~mm} \times 1 \mathrm{~mm}$ square near the center of the droplet footprint, and was plotted against the molar amount of cloxacillin and analysed using non-linear regression.

Recovery of Cloxacillin from spiked pig plasma. MMIP microspheres $(10 \mathrm{mg})$ were incubated with $5 \mathrm{ml}$ pig serum 
spiked with different concentrations of cloxacillin for 1 hour. The MMIP microspheres were then collected by magnet and washed with $5 \times 10 \mathrm{ml}$ of methanol. Cloxacillin was eluted by the addition of $5 \mathrm{ml}$ methanol:acetic acid (9:1) with probe sonication. The solution containing the eluted sample was quickly separated by magnet to avoid rebinding of the product. $1 \mu l$ of eluted sample was dried on each SERS substrate and the Raman spectrum was measured. The recovery of the extracted sample was measured by comparing the measured concentration against the known spiked sample concentration.

\section{RESULTS AND DISCUSSION}

Synthesis of MMIP microspheres. MMIP microspheres were successfully synthesized by imprinting cloxacillin in the presence of MAA, NIPAm, EDGMA and the $\mathrm{FeO}_{x}$ microspheres. As cloxacillin is considered an amphiphilic antibiotics, MAA was used to form hydrophilic interactions such as electrostatic and hydrogen bonds, while NIPAm formed mainly hydrophobic interactions with the template molecule ${ }^{24}$. For the optimized MIP layer, a template to monomer ratio of 1:6 was used for the polymerization, while the template to crosslinker ratio was kept at 1:2. The ratios are of high importance for the performance of the MIP. Larger molar ratio of crosslinker will lead to a MIP layer with increased thickness, as well as an excess formation of bulk polymer agglomerates. This in turn results in a MMIP with lower binding affinity, higher nonspecific binding, and a product which is difficult to wash. On the other hand, using a lower molar crosslinker ratio or an increase in mass of $\mathrm{FeO}_{\mathrm{x}} @ \mathrm{SiO}_{2}-\mathrm{MPA}$ microspheres can result in incomplete formation of the MIP layer.

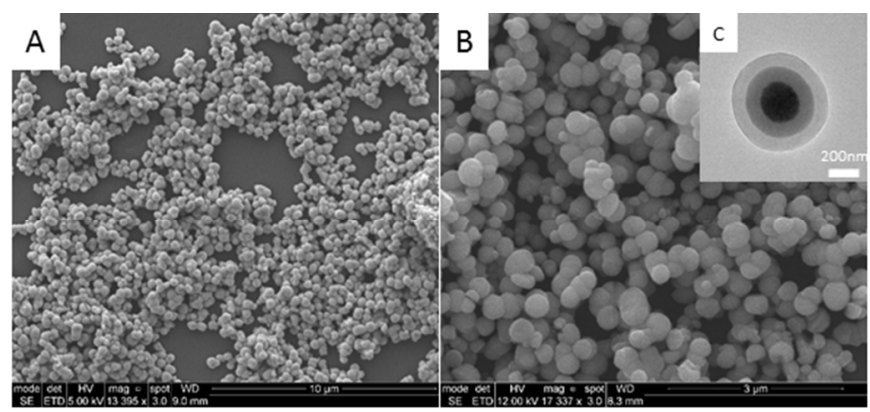

Figure 1. (A) SEM image of $\mathrm{FeO}_{\mathrm{x}}$ microspheres; (B) SEM image of $\mathrm{FeO}_{\mathrm{x}} @ \mathrm{SiO}_{2}$; and (C) TEM image of core-shell of a single MMIP microsphere, which clearly shows the three layers.

One of the biggest obstacles to the development of MMIPs is the effective removal of template after imprinting. In this study, we removed the template via a Soxhlet extractor. Although a Soxhlet extractor is quite an effective method for the removal of the template due to the repeated washes of the microspheres over 24 hours, a trace amount of residual template could remain due to encapsulation of the templates within the polymer matrix during the synthesis. In spite of this, the presence of the residual template did not affect subsequent binding experiments.
Characterization of MMIP microspheres. The morphologies of $\mathrm{FeO}_{\mathrm{x}}, \mathrm{FeO}_{\mathrm{x}} @ \mathrm{SiO}_{2}$, and $\mathrm{MMIP}$ microspheres were determined by SEM and TEM imaging. SEM images of the $\mathrm{FeO}_{\mathrm{x}}$ magnetic core (Figure 1A) are spherical with a diameter of about $230 \mathrm{~nm}$ while a size increases to around $300 \mathrm{~nm}$ for $\mathrm{FeO}_{\mathrm{x}} @ \mathrm{SiO}_{2}$ (Figure 1B). The corresponding TEM image of a MMIP microsphere shows two distinct layers surrounding the magnetic nanoparticles, indicating successive additions of the silicon layer and MIP layer (Figure 1C).

The magnetic core was made up of smaller nanoparticles of about $10 \mathrm{~nm}$ in diameter (determined from the XRD diffraction pattern). The aggregation of these nanoparticles allows for a much higher magnetic moment compared to their individual magnetic properties ${ }^{25}$. The size and zeta potential of MMIP microspheres are shown in Table S1 (Supporting Information). Upon polymerization, the thickness of the MIP layer was about $80 \mathrm{~nm}$ as determined from the TEM image.

$\mathrm{FeO}_{\mathrm{x}}, \mathrm{FeO}_{\mathrm{x}} @ \mathrm{SiO}_{2}$, and MMIP microspheres were further characterized using, IR, XRD and XPS. The IR spectra (Figure 2A) show the apparent changes in the morphology of the surface after successive modification steps.

A

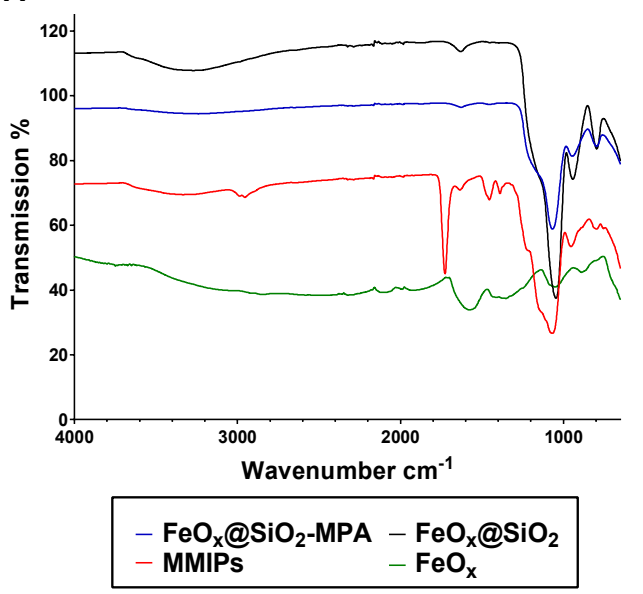

B

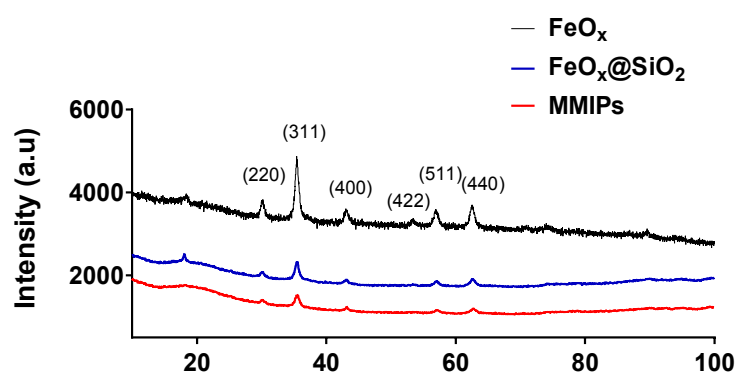

$2 \theta$ (Degrees)

Figure 2. (A) IR spectra of iron oxide, $\mathrm{FeO}_{\mathrm{x}} @ \mathrm{SiO}_{2}$, and MMIP microspheres; (B) XRD of $\mathrm{FeO}_{x}, \mathrm{FeO}_{\mathrm{x}} @ \mathrm{SiO}_{2}$, and MMIP microspheres.

Distinct IR peaks at about 953 and $1079 \mathrm{~cm}^{-1}$ corresponding to Si-O-H stretching vibration and $\mathrm{Si}-\mathrm{O}-\mathrm{Si}$ asymmetric stretching vibration respectively were observed. The peak observed at 
$807 \mathrm{~cm}^{-1}$ corresponds to Si-O-Si symmetric stretching vibration. No observed changes were observed between the IR spectra of $\mathrm{FeO}_{\mathrm{x}} @ \mathrm{SiO}_{2}, \mathrm{FeO}_{\mathrm{x}} @ \mathrm{SiO}_{2}-\mathrm{MPA}$ due to the fact that the MPA forms a monolayer which cannot be observed using the IR ATR setup. However a peak at $1732 \mathrm{~cm}^{-1}$ corresponding to $\mathrm{C}=\mathrm{O}$ confirms the presence of the MIP layer.

The XRD in the $2 \theta$ range of $10-80^{\circ}$ (Figure 2B) shows peak positions indexed to (220), (311), (400), (422), (511) and (440), confirming the spinel structure of $\mathrm{FeO}_{\mathrm{x}}$ core. Corresponding XRD spectra shows that these peaks are retained for $\mathrm{FeO}_{\mathrm{x}} @ \mathrm{SiO}_{2}$, and MMIP microspheres. VSM was used to characterize the magnetic properties of the $\mathrm{FeO}_{\mathrm{x}}$ core, $\mathrm{FeO}_{\mathrm{x}} @ \mathrm{SiO}_{2}, \quad \mathrm{MMIP}$ microspheres respectively. Saturation values obtained were 69,23 and $14 \mathrm{Am}^{2} / \mathrm{kg}$, respectively. The VSM hysteresis loops are shown in Figure 3. All three samples showed a similar shape and were symmetrical about the origin. The samples showed a behavior with a remanence and coercive field close to zero, which is a character for superparamagnetic particles. The decrease in $\sigma_{\mathrm{s}}$ observed in the $\mathrm{FeO}_{\mathrm{x}} @ \mathrm{SiO}_{2}$ and MMIP microspheres is due to the contributions of the $\mathrm{SiO}_{2}$ and MIP layers.

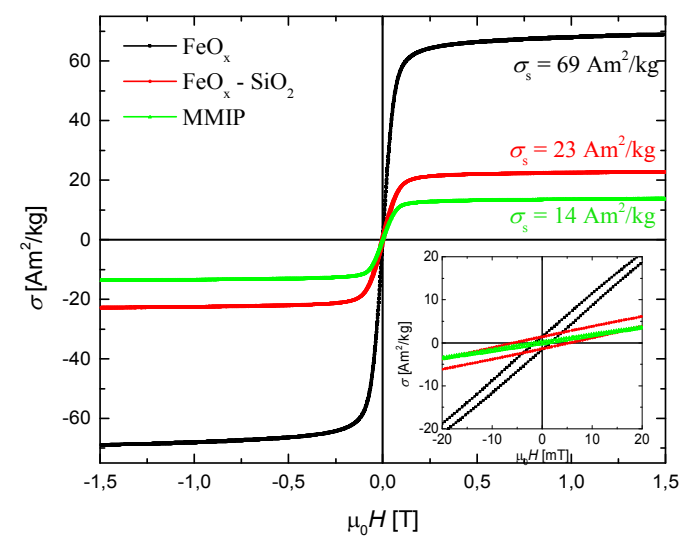

Figure 3. Magnetization curves for $\mathrm{FeOx}, \mathrm{FeOx} @ \mathrm{SiO}_{2}$, and MMIP microspheres.

Binding of cloxacillin to MMIP microspheres. The adsorption kinetics of cloxacillin to MMIP microspheres was analysed using UV spectroscopy. The difference in concentration before and after incubation was measured at different time points (1-180 minutes). The binding saturation plot (Figure 4A) showed that the binding reached a saturation point after 20 minutes, suggesting that the binding occurred rapidly. The saturation of the binding sites on the MMIP microsphere surface prevented further binding from occurring. It also suggested that the binding sites were near to the surface of the MMIP microspheres. The MNIP microspheres showed a lower affinity towards cloxacillin and also reaching a point of saturation within a few minutes due to the random distribution of functional groups on the surface. It is worth noting that the time for binding can change with different concentrations of cloxacillin.
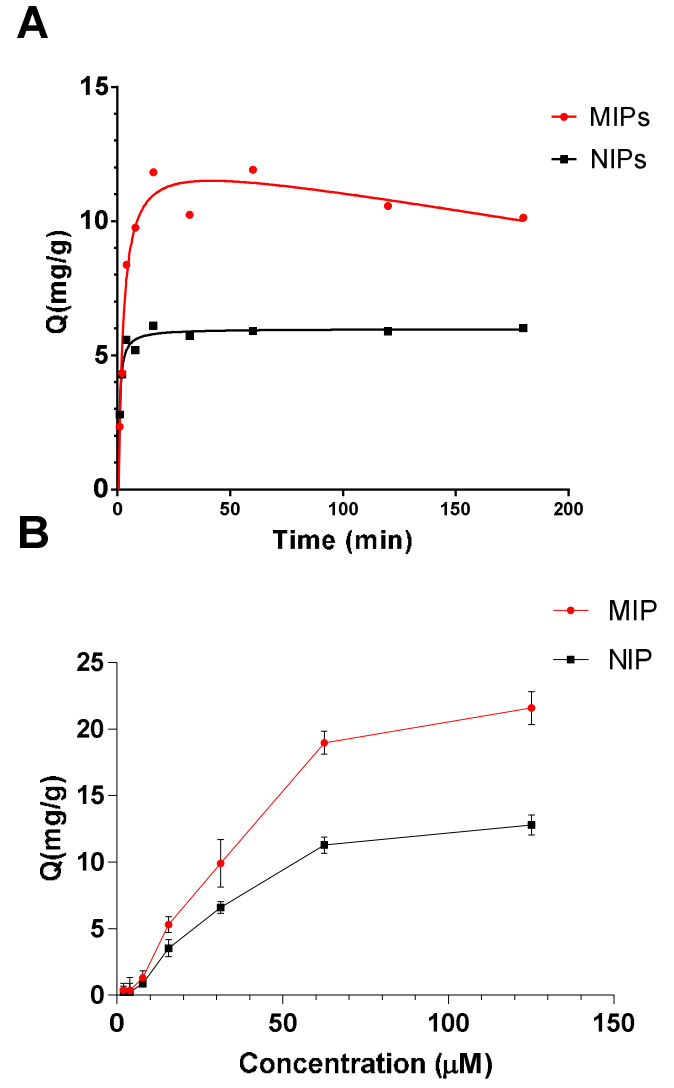

Figure 4. Binding of cloxacillin to MMIP microspheres. (A) Binding kinetics; and (B) adsorption capacity.

The binding isotherm curves (Figure 4B) showed an increase in adsorption capacity with increasing cloxacillin concentration. The MMIP microspheres showed a higher adsorption capacity compared to the MNIP microspheres, demonstrating at least a two-fold increase at the highest concentration.

The equations for determining the binding affinity and kinetics of MMIP microspheres and corresponding MNIP microspheres were discussed further in the supporting information. From the slopes and intercept of the scratchard plot (Figure S1) the values of $K_{d}$ and $Q_{\max }$ were $36 \mathrm{mg} \mathrm{L}^{-1}$ and $39.5 \mathrm{mg} \mathrm{g}^{-1}$, respectively. The linear relationship of the plot indicates that the binding sites on the surface of the MMIP were very homogenous. The maximum concentration measured was 125 $\mu \mathrm{M}$ due to saturation of UV absorbance signal at higher concentrations.

The selectivity of MMIP microspheres against other antibiotics was accessed by incubating $100 \mu \mathrm{M}$ of each antibiotic with $10 \mathrm{mg}$ of MMIP microspheres. The MMIP microspheres demonstrated a two-fold increase in binding towards cloxacillin compared to spiramycin and a five-fold increase compared to doxycycline hyclate (Figure 5). Gentamycin demonstrated very little binding towards the MMIP microspheres. MNIP microspheres demonstrated a similar binding affinity towards deoxycycline hyclate and spiramycin, which showed that their binding was from contributions of non-specific binding. The non-specific binding to the MNIP might arise from the interactions between the functional groups of spiramycin and the NIP 
polymer material, which is difficult to completely avoid. However, all the antibiotic residues tested can be easily distinguished based on the differences in the corresponding fingerprint SERS spectra, which overcomes the current limitations of MIP technology.

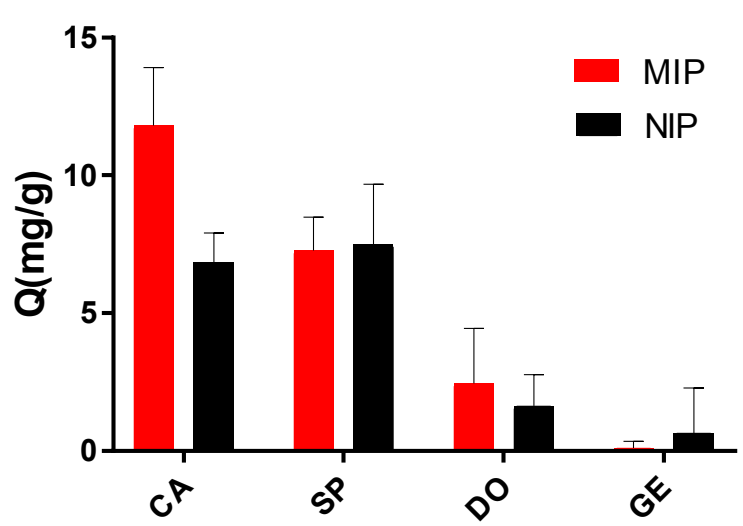

Figure 5. Selectivity of MMIP microspheres for Cloxacillin (CA), Spiramycin (SP), Doxycycline hyclate (DO), and Gentamycin sulfate (GE).

The nanopillar SERS substrate. Wafer-scale metal capped silicon nanopillars were fabricated using a lithography-free process $^{20-22}$. The fabricated nanopillars were flexible and could lean towards their nearest neighbors after the deposited analyte solution evaporated, creating self-assembled SERS hot spots in which the analyte molecules were located. SERS enhancement factors of above $10^{8}$ have been achieved with good uniformity $^{28}$. Applications such as detecting nerve gases have been realized on such substrates with high sensitivities ${ }^{26}$. The fabrication procedure is fast, repeatable and does not require any lithographic steps. The low fabrication cost enables these SERS substrates to be used as low cost and single use consumables. The SERS substrates used for the detection of cloxacillin were gold capped silicon nanopillars, shown in Figure 6. To obtain optimal SERS spectra, leaning and clustering of the nanopillars are necessary. Figure $\mathbf{6 C}$ shows the clusters of the nanopillars.

Upon drying of the analyte solution deposited onto the substrate, surface tension pulled the vertical standing nanopillars to lean toward their nearest neighbors, creating self-assembled SERS hot spots at their gap junctions ${ }^{21}$. The Raman scattering signals of the analytes located inside or near these hot spots were boosted electromagnetically, and scaled with the widely known $\mathrm{E}^{4}$ relationship ${ }^{27}$.

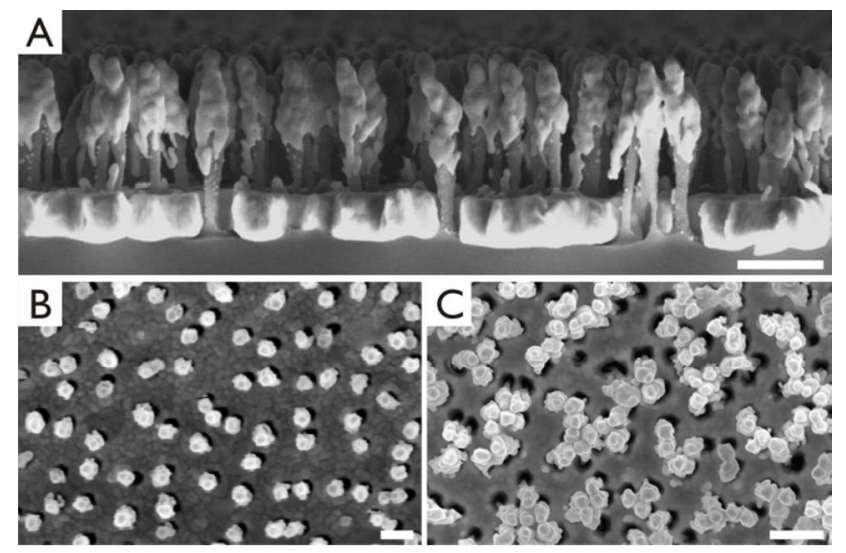

Figure 6. (A) and (B) A cross-sectional and a top-view SEM image of the vertically standing gold capped silicon nanopillars, respectively. The e-beam assisted deposition of gold resulted in the formation of gold caps at the apex of the Si NPs, along with a gold film on the underlying silicon surface; (C) A top-view SEM image showing the clusters of the gold capped silicon nanopillars. The ability for the vertical nanopillars to lean towards their neighbors and create hot spots in the gap junctions with strong local optical fields qualifies them for a very effective SERS-based sensor. Scale bars: $400 \mathrm{~nm}$.

Detection of Cloxacillin using SERS. SERS was used to measure the Raman spectra of different concentrations of cloxacillin in buffer (methanol:acetic acid $=9: 1$ ) to determine the limit of detection under optimum conditions. Samples were dried on the nanopillar substrates before measuring.

Spectra are shown in Figure 7A. At zero concentration, five prominent peaks were shown on the spectrum at 1534, 1372, 1231, 931 and $807 \mathrm{~cm}^{-1}$. Their corresponding molecular fingerprint matched the Raman fingerprint of acetic acid despite a red shift, indicating the adsorption of acetic acid to the gold surface $^{28}$. With increased concentration of cloxacillin, a number of peaks emerged on the spectra with gradually increased intensities, as shown in Figure 7A. The limit of detection for cloxacillin was determined to be 7.8 pmol under optimal conditions.

Quantitative analysis is essential to obtain a reliable calibration standard. However, achieving good quantification is a big challenge in SERS ${ }^{29}$. Here, to overcome this challenge, acetic acid was used as SERS internal standard ${ }^{30}$. Particularly, the SERS intensity at $931 \mathrm{~cm}^{-1}$ corresponding to the C-C stretching band of acetic acid ${ }^{28}$, was used as a divisor to normalize the SERS intensity of cloxacillin at its most prominent peak, i.e., $1034 \mathrm{~cm}^{-1}$. This approach was highly feasible, since (i) the selected two bands were well separated from other peaks, (ii) these two bands had high Raman cross sections, and (iii) acetic acid was present due to the nature of the sample itself, and no additional sample treatment was needed. 

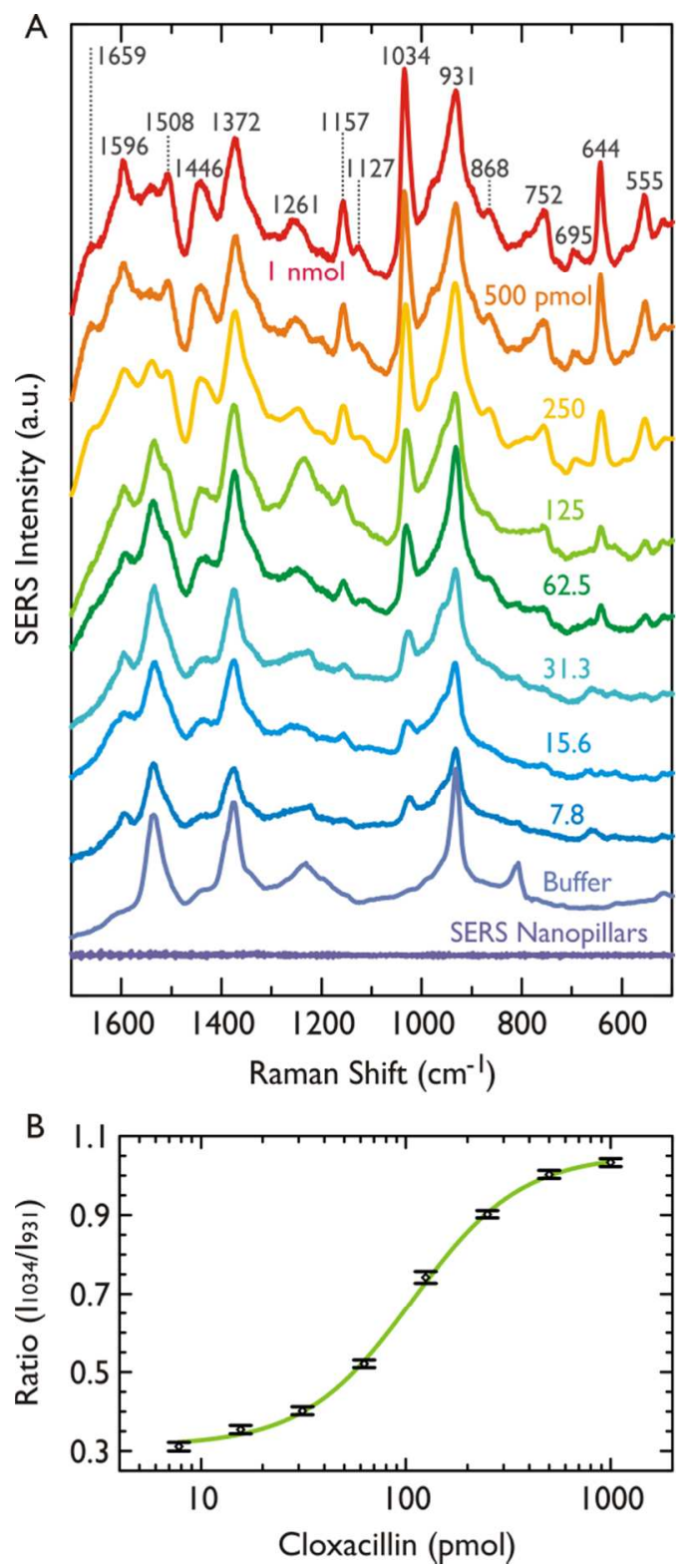

Figure 7 A) SERS spectra for different amount of cloxacillin deposited on the nanopillar substrate. SERS intensities were normalized to improve visual clarity. (B) The non-linear calibration plot $(n=3)$. Each ratio of intensity was averaged over 121 spectra obtained in a $1 \mathrm{~mm} \times 1 \mathbf{m m}$ droplet footprint.

Furthermore, quantification using acetic acid as SERS internal standard had the following advantages: (i) signal variations caused by instrumental factors such as laser power, alignment of sample and collection optics were removed; (ii) nonuniform signals over the substrate due to different surface wetting and drying properties were eliminated; and (iii) the uneven signal enhancement on account of the non-uniform nature of the plasmonic near field was compensated. All of the above contributed to more reliable and reproducible signals, turning quantitative SERS into reality.

To quantify and establish SERS calibration standards for cloxacillin using the nanopillar substrate, the intensity ratio of the 1034 and $931 \mathrm{~cm}^{-1}$ peak after baseline correction was averaged over a $1 \mathrm{~mm} \times 1 \mathrm{~mm}$ droplet footprint. The averaged ratio against the molar amount of cloxacillin is plotted in Figure 7B. Examples of SERS maps are shown in Figure 8. It can be seen that SERS uniformity and reproducibility were improved dramatically after normalization using an internal standard.

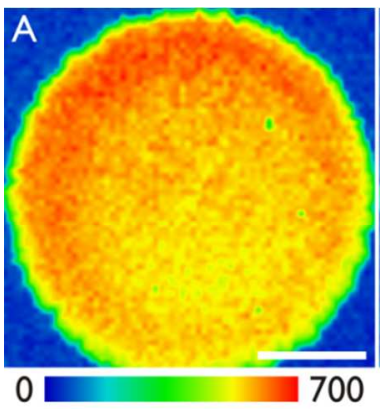

SERS Counts

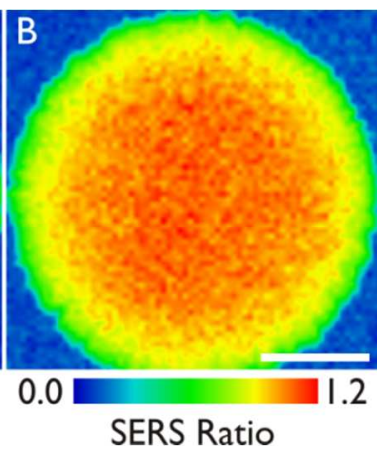

Figure 8. SERS images of 500 pmol cloxacillin obtained on the gold nanopillar substrate (A) before and (B) after normalization. $1 \mu \mathrm{l}$ sample was added to the SERS substrate. SERS intensity at $931 \mathrm{~cm}^{-1}$ of acetic acid was used as internal standard to normalize the SERS intensity of cloxacillin at $1034 \mathrm{~cm}^{-}$ 1. Scale bars: $2 \mathrm{~mm}$.

Next, SERS detection and quantification of cloxacillin in pig plasma was conducted. The use of pig serum as the matrix has several advantages over liver and muscle samples which include more facile sampling and pre-treatment of the sample prior to analysis. Pig serum samples were prepared by spiking different concentrations of cloxacillin standard and incubating at room temperature.

After incubation, the microspheres were washed to remove all traces of the serum matrix effects. The cloxacillin residues were eluted by incubating the microspheres with methanol:acetic acid (9:1) mixture and separated by magnet. $1 \mu 1$ of each sample was added to the SERS substrate as described for the calibration standards. The recorded spectra in Figure S2 (supporting information) show similar spectral fingerprints to those shown in Figure 7A, indicating that the extraction did not introduce additional SERS background signal. As shown in Table 2, recoveries of pig plasma samples spiked with cloxacillin $(15.6$ - $500 \mathrm{pmol})$ were from 85 to $126 \%$ with relative standard deviations (RSDs) ranging from 3.9 to $18.7 \%$.

Table 2 Percentage recoveries and RSD of cloxacillin from spiked pigs serum samples $(n=3)$.

\begin{tabular}{cccc}
\hline Measured & & \\
Spiked $(\mathrm{pmol})$ & $(\mathrm{pmol})$ & \% recovery & \%RSD \\
\hline 500 & 505.5 & 101.1 & 18.7 \\
\hline 250 & 240.3 & 96.14 & 8.8 \\
\hline 125 & 132.2 & 105.8 & 10.3 \\
\hline 31.25 & 26.4 & 84.5 & 3.9 \\
\hline 15.6 & 19.6 & 126 & 11.8 \\
\hline
\end{tabular}


The highest recovery shown at the lowest spiked concentration of 15.6 pmol might be due to the residual matrix effects of the plasma which became more apparent at low concentrations. The lack of SERS response from the non-spiked sample demonstrated that there was little or no template leakage from the MMIP, confirming adequate template removal during the Soxhlet extraction procedure. The results suggested that the combination of MMIP-based sample preparation and SERSbased detection was an effective method for analyzing antibiotic residues in complex food matrices.

\section{CONCLUSION}

In this study, we demonstrated the combined use of MMIP microspheres and SERS for the detection of cloxacillin in pig plasma. Core-shell MMIP microspheres were successfully synthesized and characterized. The MMIP microspheres demonstrated the ability to rapidly and selectively extract cloxacillin from pig plasma with minimal matrix effects. SERS substrates with gold capped silicon nanopillars were fabricated, and sensitive detection of cloxacillin was demonstrated. Particularly, quantitative SERS was achieved by using acetic acid as an internal standard. The analytical method brings together the facile manipulation of magnetic microspheres, the high selectivity and binding capacity of MIPs, and the high sensitivity of SERS detection. In addition, attributed to the fast analysis time, low cost and the possibility for miniaturization and integration, the developed method holds great potential for applications in point-of-need settings.

\section{ASSOCIATED CONTENT}

\section{Supporting Information}

Kinetics isotherm scratchard plot for MMIP microspheres; size and zeta potential of MMIP microspheres; cloxacillin SERS spectra of extracted samples.

The Supporting Information is available free of charge on the ACS Publications website.

\section{AUTHOR INFORMATION}

\section{Corresponding Authors}

*Email: sun.yi@nanotech.dtu.dk. Phone:+45 45256319.

*Email: kaiwu@nanotech.dtu.dk. Phone:+45 45255787.

\section{Author Contributions}

The manuscript was written through contributions of all authors. / All authors have given approval to the final version of the manuscript. / $\$$ These authors contributed equally.

\section{ACKNOWLEDGMENT}

This work was financially supported by the Villum Fonden, Denmark, Project No. 13153; and the IDUN project, The Danish National Research Foundation, Project DNRF122.

\section{REFERENCES}

(1) Council of the European Union. 2009, No. 4.

(2) Jank, L.; Martins, M. T.; Arsand, J. B.; Hoff, R. B.; Barreto, F.; Pizzolato, T. M. Food Addit. Contam. Part A 2015, 1-10.

(3) Blanchaert, B.; Poderós Jorge, E.; Jankovics, P.; Adams, E.; Van Schepdael, A. Chromatographia 2013, 76 (21-22), 1505-1512.

(4) Song, J. Y.; Hu, S. J.; Joo, H.; Hwang, J. B.; Kim, M. O.; Kang,
S. J.; Cho, D. H. World Acad. Sci. Eng. Technol. 2011, 57 (9), 809-811.

(5) Posyniak, A.; Zmudzki, J.; Niedzielska, J. Anal. Chim. Acta 2003, $483(1-2)$.

(6) Cháfer-Pericás, C.; Maquieira, Á.; Puchades, R. TrAC Trends Anal. Chem. 2010, 29 (9), 1038-1049.

(7) Chen, L.; Li, B.; Nie, C.; Kong, J.; Liu, X.; Wen, T.; Yuan, L.; Dai, P.; Lin, Y.; Zhou, X.; Mei, S.; Thang, S. Anal. Methods 2012, 4 (9), 2613.

(8) Urraca, J. L.; Chamorro-Mendiluce, R.; Orellana, G.; MorenoBondi, M. C. Anal. Bioanal. Chem. 2016, 408 (7), 1843-1854.

(9) Díaz-Bao, M.; Barreiro, R.; Miranda, J. M.; Cepeda, A.; Regal, P. J. Anal. Methods Chem. 2015, 2015, 1-8.

(10) Kahraman, M.; Mullen, E. R.; Korkmaz, A.; WachsmannHogiu, S. Nanophotonics 2017, $O(0)$.

(11) Kamra, T.; Zhou, T.; Montelius, L.; Schnadt, J.; Ye, L. Anal. Chem. 2015, 87 (10), 5056-5061.

(12) Lan, L.; Yao, Y.; Ping, J.; Ying, Y. Biosensors and Bioelectronics. 2017, pp 504-514.

(13) Zheng, J.; He, L. Compr. Rev. Food Sci. Food Saf. 2014, 13 (3), 317-328.

(14) Feng, S.; Hu, Y; Ma, L.; Lu, X. Sensors Actuators, B Chem. 2017, 241

(15) Hu, Y.; Lu, X. J. Food Sci. 2016, 81 (5).

(16) Gao, F.; Hu, Y.; Chen, D.; Li-Chan, E. C. Y.; Grant, E.; Lu, X. Talanta 2015, 143.

(17) Hu, Y.; Feng, S.; Gao, F.; Li-Chan, E. C. Y.; Grant, E.; Lu, X. Food Chem. 2015, 176.

(18) Gao, F.; Feng, S.; Chen, Z.; Li-Chan, E. C. Y.; Grant, E.; Lu, X. J. Food Sci. 2014, 79 (12), N2542-N2549.

(19) Guo, Z.; Chen, L.; Lv, H.; Yu, Z.; Zhao, B. Anal. Methods 2014, $6(6), 1627-1632$.

(20) Schmidt, M. S.; Hübner, J.; Boisen, A. Adv. Mater. 2012, 24 (10), OP11-OP18.

(21) Wu, K.; Rindzevicius, T.; Schmidt, M. S.; Mogensen, K. B.; Xiao, S.; Boisen, A. Opt. Express 2015, 23 (10), 12965-12978.

(22) Wu, K.; Rindzevicius, T.; Schmidt, M. S.; Mogensen, K. B.; Hakonen, A.; Boisen, A. J. Phys. Chem. C 2015, 119 (4), 20532062.

(23) Hassanain, W. A.; Izake, E. L.; Schmidt, M. S.; Ayoko, G. A Biosens. Bioelectron. 2017, 91, 664-672.

(24) Ruso, J. M.; Attwood, D.; Garcia, M.; Taboada, P.; Varela, L. M.; Mosquera, V. Langmuir 2001, 17 (17), 5189-5195.

(25) Han, S.; Li, X.; Wang, Y.; Su, C. Anal. Methods 2014, 6 (9), 2855.

(26) Hakonen, A.; Rindzevicius, T.; Schmidt, M. S.; Andersson, P. O.; Juhlin, L.; Svedendahl, M.; Boisen, A.; Käll, M.; Zhang, R.; Sun, H. B.; Zaccaria, R. P.; Perozziello, G.; Tirinato, L.; Toma, A.; Cuda, G.; Cingolani, R.; Fabrizio, E. Di. Nanoscale 2016, 8 (3), 1305-1308

(27) Schatz, G. C.; Duyne, R. P. V. D. Surface-enhanced Vib Spectrosc. 2002, 1-16.

(28) Nakabayashi, T.; Kosugi, K.; Nishi, N. J. Phys. Chem. A 1999 103 (43), 8595-8603.

(29) Shen, W.; Lin, X.; Jiang, C.; Li, C.; Lin, H.; Huang, J.; Wang, S.; Liu, G.; Yan, X.; Zhong, Q.; Ren, B. Angew. Chemie - Int. Ed. 2015, 54 (25), 7308-7312.

(30) Bell, S. E. J.; Sirimuthu, N. M. S. Chem. Soc. Rev. 2008, 37 (5), 1012 . 